\title{
Effects of the stochastic resonance for face detection on dark and illumination variant images
}

\author{
Bikash Lamsal*, Noriko Kojima, Naofumi Matsumoto \\ Department of Information and Production Engineering, Ashikaga Institute of Technology \\ Ohmae cho 268-1, Ashikaga, Tochigi, 326-8558 Japan \\ *Corresponding Author: bikashaitjp@ gmail.com
}

\begin{abstract}
This paper concerns with stochastic resonance for the face detector that detects the face from the dark static images under different illumination conditions. The face candidates of the dark images are very hard to identify as they are not clearly visible. In this paper, we have designed an algorithm by combining the stochastic resonance, and our high performance face detector presented previously. We are applying our SR for image processing in a dark image for extracting the face candidates from the image to make them visible. The Gaussian white noise is applied to the dark image and they are summed up in a series for extracting the image candidates from the dark images and for making them visible. This process is also known as the summing network of the stochastic resonance noise. The dark image once made visible is applied to our face detector for detecting the presence of the face in an image. The use of stochastic resonance in a face detection algorithm for the static dark images reflects the novelty of our paper. We performed various experiments on various dark images under different illumination conditions and confirmed the effectiveness of SR for detecting the faces in a dark image.
\end{abstract}

Keywords: Dark image, Face detection, Image enhancement, Stochastic resonance.

\section{Introduction}

The physiological biometric identifier that is widely used in human recognition is the face. It is recognized only after the face detection. Face detection is done either on the static images or on the video images. We are using the static images in our face detection process. We developed a "high performance face detector" ${ }^{(1)}$ for detecting the face in a static image, but the face detection rate has not reached $100 \%$ due to the presence of different environmental conditions in the images. Among them is dark images with the illumination variants. Face detection is especially difficult in this case because the face candidates are not properly visible.

In order to overcome this environmental difficulties, the contrast adjustment process has been applied by T. Sim and T. Kanade ${ }^{(2)}$ for the different illuminating examples, whereas S. Hayashi and O. Hasegawa worked on the low resolution image ${ }^{(3)}$. These processes work properly on some images, but still there are some illumination variant conditions which are not satisfied with these methods. To overcome the problem of dark and illumination invariant images, we adopted the stochastic resonance technique ${ }^{(4)}$ on the image that is not satisfied by the contrast adjustment process. We use the stochastic resonance (SR) as preprocessing for extracting the face candidates from a dark and illumination variant image. SR is expected to make dark images under illumination conditions visible and clear. The images after pre-processed, then passed to our high performance face detector for detecting the presence of a face in an image.

$\mathrm{SR}$ is a phenomenon in which a response of a system is enhanced by adding the Gaussian white noise ${ }^{(5)}$. It can also be known as the counter-intuitive phenomenon where the presence of noise in a non-linear system is essential for optimal system performance.

In this paper, we test our algorithm on two different techniques of SR, one is the threshold technique ${ }^{(6)}$, where the noise is tuned along with a threshold. In this case, after the Gaussian white noise has been added to the dark and the illumination variant image. The threshold operates on each pixel of that image. The results obtained by using this technique are quite complicated because of the inappropriate 
tuning as auto-tuning process is difficult.

The second technique is the summing SR technique or SR without tuning ${ }^{(7)}$. This technique is probably being used in signal processing, but in this paper, we slightly modify this for application to image processing.

We are applying Gaussian white noise on a dark and illumination variant image and sum it up with the original image. The resulted image is also summed up again with the previous images for several times until the images are not enhanced. We are setting the threshold value at zero in our algorithm. Because in the case of image processing, the pixel series of the images are calculated instead of time series. This makes it possible to protect the image from losing the special features which are necessary to detect faces in an image.

This idea is a new approach to pre- processing for face detection. It really reflects the novelty of our paper. The images after being enhanced and made visible are sent to our high performance face detector for detecting the presence of the faces in an image. We clarify the effectiveness of our technique by several experiments.

It was very difficult for us to find a database with many dark and illumination variant images for performing our experiment. Therefore, we developed our own database by taking some pictures in dark places under different illumination conditions. Also, we used a few illumination variant images from the INRIA Graz-01 (8), and FDDB database (9) which could not be detected by our high performance face detector ${ }^{(1)}$.

\section{Stochastic Resonance for Image processing}

There are two different techniques for SR, i.e., "threshold phenomenon" and "summing SR" technique. The summing SR technique is also known as "SR without tuning" by Collins et al. ${ }^{(7)}$. In case of image processing, we have developed "our SR technique for image processing" from the idea of the SR without tuning.

\subsection{Original SR technique}

SR was first presented by Benzi ${ }^{(3)}$. The process of the original SR technique is shown in figure 1.

A white noise is added to the input image and then the threshold is applied to the image. In this process, the threshold has to be tuned along with the applied noise for getting an appropriate result. The auto tuning is quite a complicated task. Therefore, we have to tune a noise level and a threshold manually. The experiment performed on a dark image by using this process is shown in figure 2 and

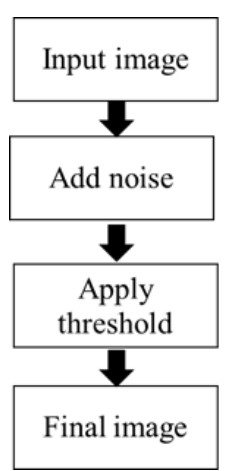

Fig.1. Process of the original SR technique.

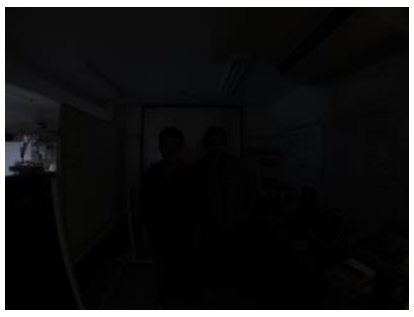

(a)

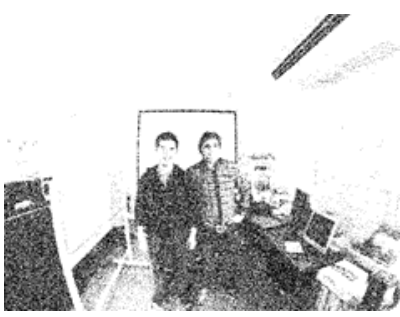

(c)

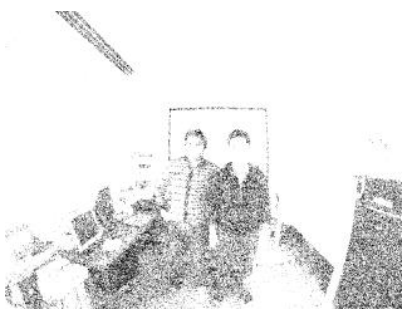

(b)

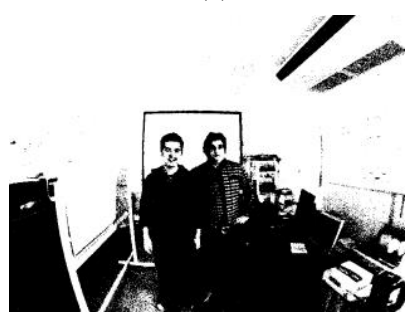

(d)
Fig.2. Example of images improved by the original SR.

(a) Original dark image. (b) Noise level $=1$, threshold value $=1$. (c) Noise level=2, threshold=3. (d) Noise level=4, threshold $=4$.

Table 1. Face detection results for Fig.2 (a) by the original SR.

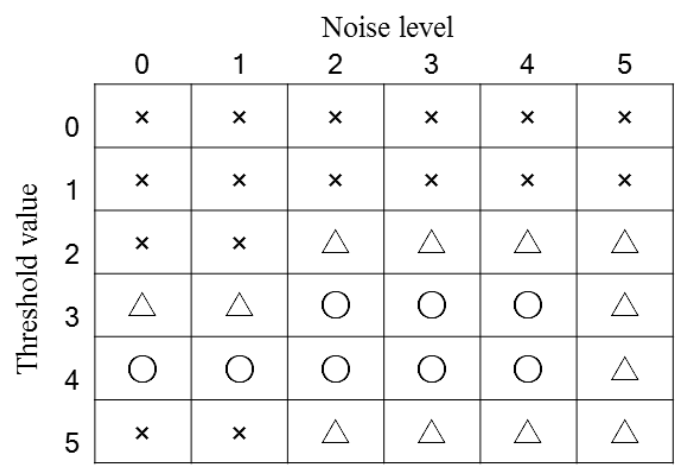

the matrix showing the face detection under different noise level and the threshold value is shown in table 1 . In table 1, " $\bigcirc$ " represents the true face detection, " $\times$ " represents the false and " $\Delta$ " represents only one face out of two faces is detected. The table shows that the noise level and the threshold value have to be tuned properly in order to get the 
proper face detection. Especially, there is a very important role of the threshold in this SR type. As can be seen from this table, the face detected area is very narrow, so we do not adapt it in our proposed algorithm.

\subsection{SR without tuning}

The "SR without tuning" or the "summing network" idea was developed by Collins et.al ${ }^{(7)}$. This idea was first applied as a summing network of the identical excitable units related to the signal interference devices. As we are doing our research based on image processing, we will follow the PDM (Pulse density modulation) ${ }^{(10)}$ in our technique. Figure 3 shows the flow of our adopted SR. One of experiment results performed on dark images by using this technique is shown in figure 4.

The Gaussian white noise is first applied to an input signal $X$ sequentially. Then a threshold value is applied to each result. Finally, the noise added signals are summed up in a series to obtain the result in terms of $\hat{\boldsymbol{Y}}$.

In figure 4, the "SR without tuning" process have been applied on a dark image and we get the final enhanced image as shown in figure $4 \mathrm{~b}$. In this case, two faces are detected from this image by using our high performance face detector. However, there are some cases that the face detection is not possible by using this technique. We will describe about those cases in chapter 4 .

\subsection{Our SR technique for image processing}

We developed this idea from the "SR without tuning" by Collin's et al. as shown in figure 5. However, the threshold value is kept at constant zero in our technique.

In our process, we apply the Gaussian white noise in an image, setting the threshold value at constant zero. The noise value is normalized from 0-255 range instead of $0-1$ because the image is calculated in the pixel value instead of time. Then we sum up the white noise applied images for several times in a series. If we apply a threshold value larger than zero, the special features of the face degrades. An image showing one of the experimental results of this technique is shown along with its histogram in figure 6 (b). Comparing two histograms of figure 4 (b) and 6 (b), the pattern of the histogram obtained by using our SR technique for image processing is clear and standard.

\subsection{Why "our SR technique for image processing"?}

In our SR technique for image processing, we can apply a default noise level in a dark image and sum up the noise added image in a series. The threshold value is kept at 0 , which means that we don't need any tuning of the Gaussian white noise and the threshold in our process. This is our novelty and a new approach in the field of image processing.

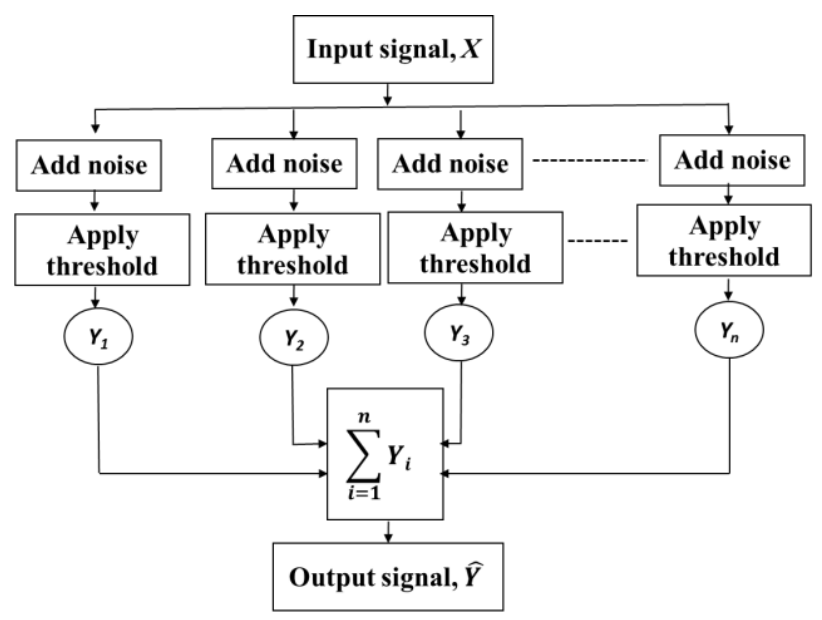

Fig.3. SR without tuning (summing network).

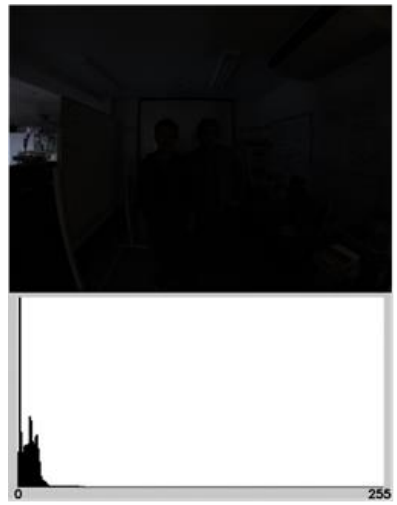

(a) Original dark image.

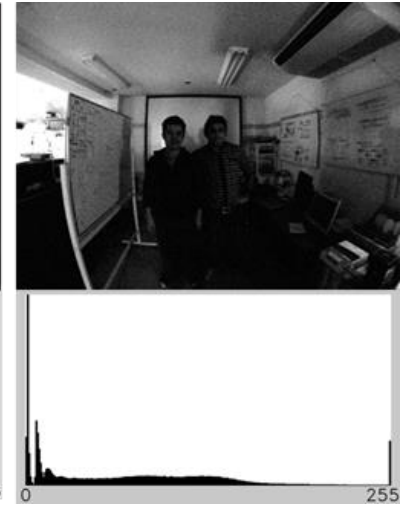

(b) Enhanced image.
Fig.4. Example using SR without tuning method.

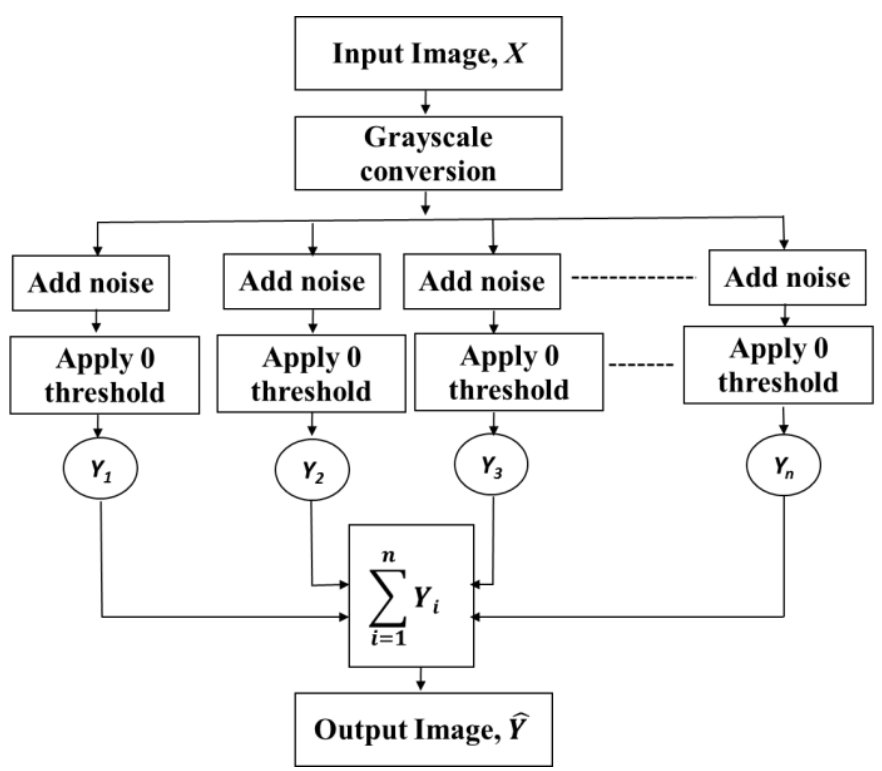

Fig.5. Our SR technique for image processing. 


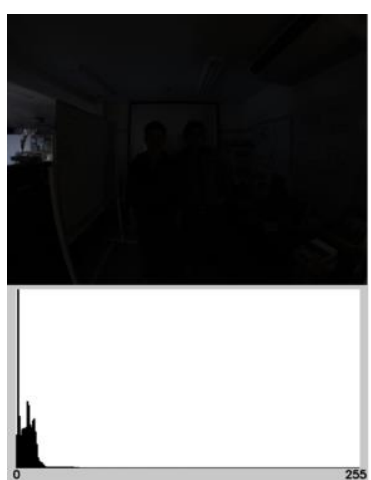

(a) Original dark image.

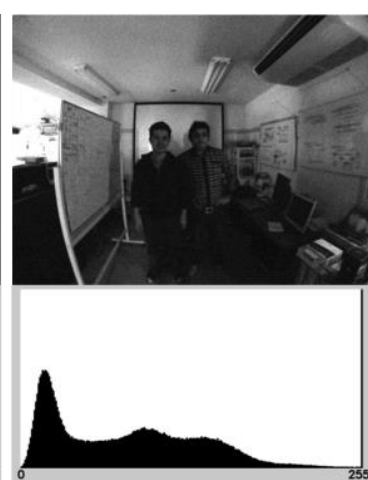

(b) Enhanced image.

Fig.6. Effect of our SR technique (same example of Fig.4).

In comparison with the "original threshold technique" and the "SR without tuning" process, our SR technique gives the best experimental result and is easy to implement. We do not need different noise level and threshold values for developing SR technique in our process. We can just apply a noise value in a single image and then sum up the same image in a series for several times with a threshold value constant 0 , which gives us a required output image.

In comparison to the "SR without tuning" process, the result is quite similar to our proposed algorithm. However, in case of "SR without tuning", we need to apply the noise levels in an image. It means that the noise has to be tuned in this process, although the threshold value is kept constant but not zero, which may lead to a loss in feature of the face present in the image as shown in the histogram of figure $4 \mathrm{~b}$ and $5 \mathrm{~b}$.

Our SR technique for image processing does not lose any pixel data and image characteristics. This method is quite easy and simple to implement SR theory for face detection.

\section{Proposed algorithm}

In our current algorithm, we are dividing the process into two parts. The first part reflects the image enhancement process and the second part reflects the face detection process. The structure of our proposed algorithm is shown in figure 7.

\subsection{Image enhancement phase}

The input image is first converted to a grayscale image in our process because, it is easy to apply SR in grayscale image in compared to the RGB image. The image after converting to grayscale image is then passed to our SR technique for image processing. In this process, the Gaussian

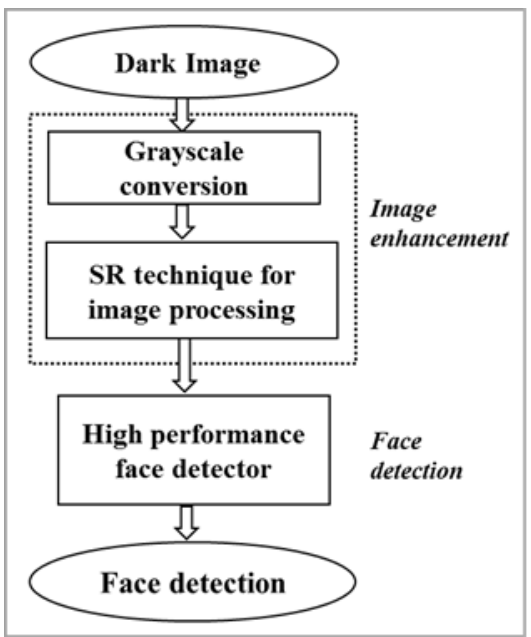

Fig.7. Proposed algorithm for face detection in dark image.

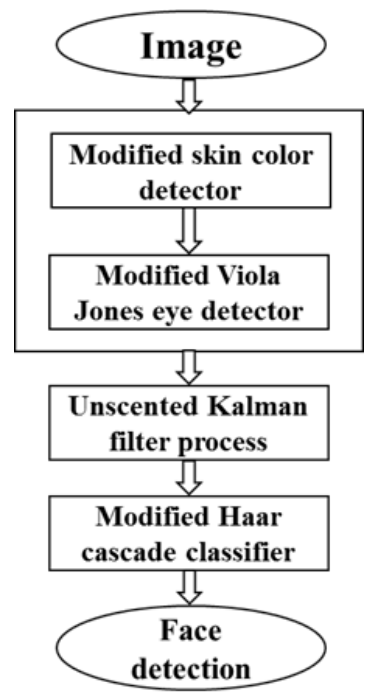

Fig.8. Our face detection algorithm (from Ref.1).

white noise is applied to an image with the threshold value at constant 0 . The image is then summed up in a series by itself for several times until the image becomes visible as shown in figure 3 . However, the threshold value is kept zero in our process because the summation is performed in the pixel series instead of time series in our process. The important feature of images or faces are not lost if we keep the threshold value at 0 constant in case of image processing.

\subsection{Face detection phase}

The main target of our current research is the detection of faces on the dark and illumination variant image. For detecting faces from an image we need a face detector. We are using our high performance face detector as shown in figure 8 . In our process, once the image is enhanced by 
applying the SR technique for image processing, we are able to apply our high performance face detector on the enhanced image and to detect the presence of faces from the given image.

\section{Experimental results}

\subsection{Preparation for experiments}

In order to validate our proposed algorithm, we performed some experiments on different dark images under different illumination conditions. Also, we compared our process with different types of SR techniques and we draw matrix tables like table 1 and pictorial charts like figure 8 .

We developed a $\mathrm{C}$ programming code for checking the efficiency of different SR techniques and draw a matrix table showing for the possibility of the face detection on different images. We developed our system by using the Visual studio 2010 and the Open CV library on PC (i7-3770, 3.40 GHz, 8GB main memory and Windows 8.1, 64 bit OS). Our system indicates the detected face in a red square box.

\subsection{Databases used}

We were doing our research on dark images under different illumination conditions. It was quite difficult for us to collect the dark images from several facial databases so we developed our own database for dark image by taking some pictures under different illumination conditions. We also downloaded some images from the internet with the permission of the author. We selected a few illumination variant images from the INRIA Graz-01 (8) database and FDDB database ${ }^{(9)}$.

\subsection{Evaluation of different image cases}

The pictorial chart showing the iteration of our proposed algorithm and the face detection result is shown in figure 9 where " $n$ " reflects the iteration number of our SR process. Here, we show the four different cases under different illumination conditions being enhanced at $n=1$ and the final iteration, where the face detection is possible. The noise value is set at 1 for all the four cases. The case 1, 2 and 3 images are downloaded from the internet with the author's permission and the case 4 image is taken by ourselves for the research purpose. These four images are the condition where the face detection is not possible by the contrast adjustment process. To show the changes of an image after applying the summation series, we show a histogram of an image along with a summed image. The dark area of each image is near to the 0 gradations and the bright, or white area is near to 255 gradations. The area of each histogram is set to the same value for all the images.

The case 1 in figure 9 was taken in a dark room with the focus light. The image is enhanced by applying the Gaussian white noise level 1 and our SR summation series for three time. The three faces are detected with the three times SR iteration by using our proposed face detector ${ }^{(1)}$. Changes in the brightness of each image are represented by the histogram along with an image

The case 2 image was downloaded from PhotoJosh, taken outside at night in front of the sparkling light. In this case also the face is detected after applying the noise level 1 and 3 time iteration of our SR technique. The face in this case is slightly rotated, but our face detector detects the face up to 90 degree rotation, so this case was satisfied by our proposed face detector. This case is not satisfied by both of the SR techniques except our SR techniques.

The case 3 image was taken at night using the focus light. Due to the presence of excess brightness in the image, the image was first inverted before applying our SR technique. In this case also, the face is detected after applying the noise level 1 and three time iteration of our SR technique. In this case also, one face is slightly rotated, but our proposed face detector was able to detect both faces in the image.

Similarly, the case 4 image was at night in front of the illuminating light. After applying the summation series for ten times, we were able to detect the presence of a face in the image, but along with some false positives as shown in a red box. This type of images is a quite difficult case for the face detection because the faces can be seen by our human eyes but due to the reflection of light in the face, the face detection system cannot detect the face in the image. In this case, the histogram is drawn in different scale for the clear visibility of the histogram. The contrast adjusted image seems to be quite acceptable, but due to the mixture of light, the contrast adjusted image is not detected by our proposed face detector.

The image shown in figure 10 is taken from the INRIA Graz-01 DB ${ }^{(8)}$. This image is taken inside a tunnel for a bright illumination variant image. The face detection is quite difficult in such types of images, as the images are taken in a bright background with a shadow on the face. We tried different types of face detectors for detecting the faces in this image, but we were unable to detect the face. Even our proposed face detector was unable to detect the face in this image. So, we apply the contrast adjustment process in this image and as a result, we were able to detect the presence of face in this image. 

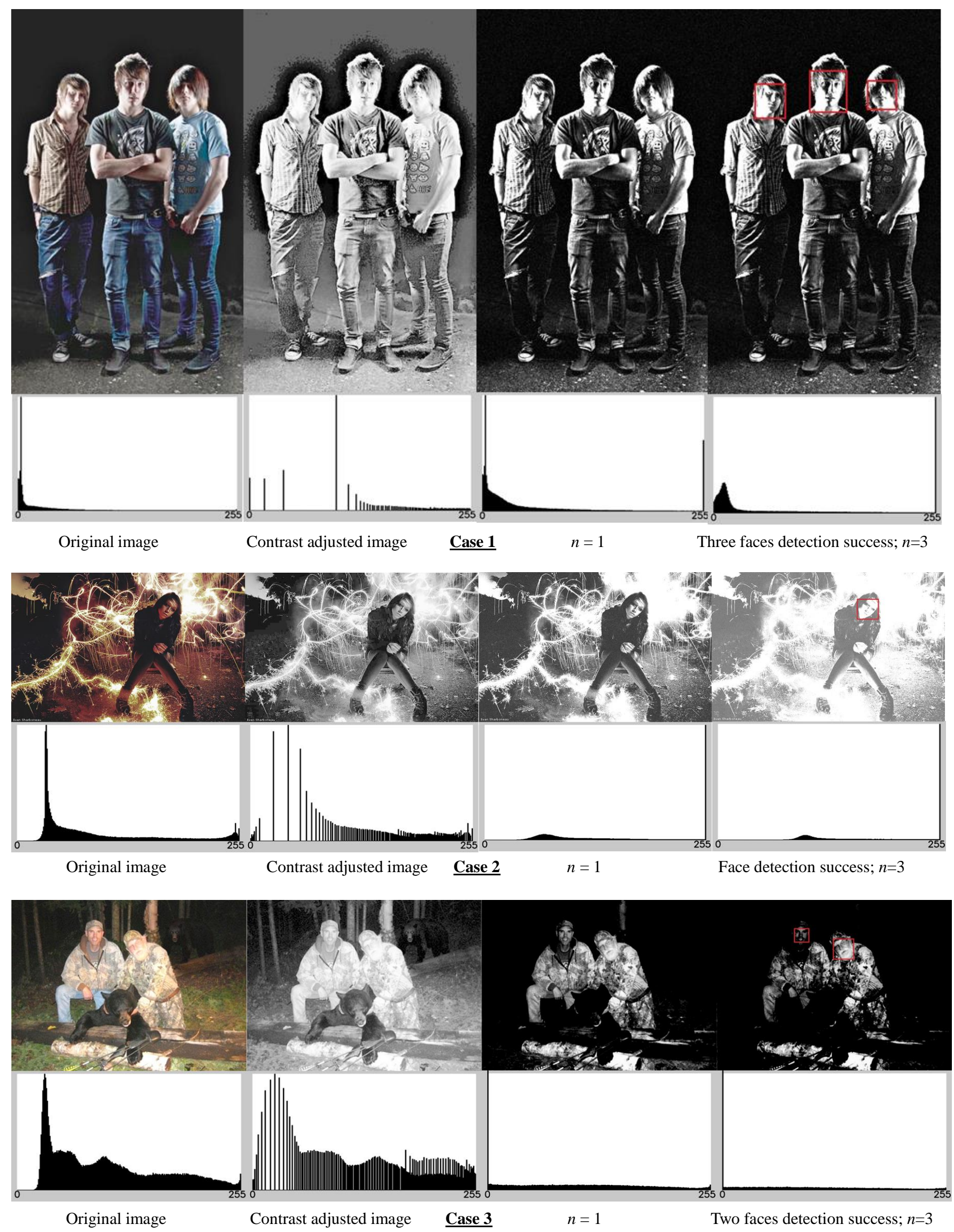

Fig.9. Evaluation of our proposed algorithm on four different dark images along with each histogram.

(Continue on next page) 

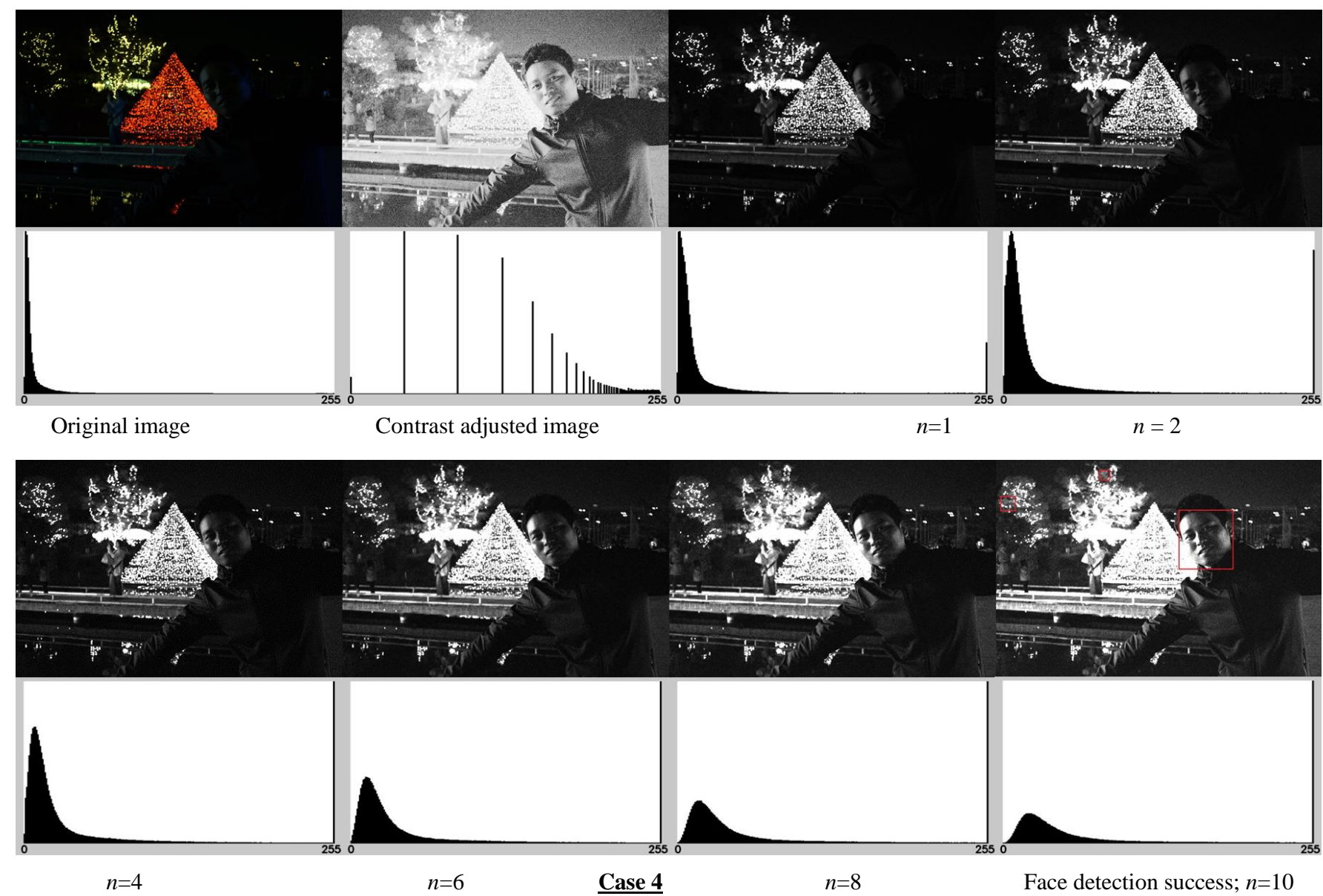

Fig.9. Evaluation of our proposed algorithm on four different dark images along with each histogram.

\subsection{Discussions}

We performed various experiments under different cases related to dark and illumination variant images. Our SR technique is designed to work on dark and illumination variant images. The proposed algorithm can detect faces with different scales even they are taken in a dark environment. The illumination variant images that were not detected by using the contrast adjustment process along with the face detector ${ }^{(1)}$ are now detected by using our proposed algorithm using the SR technique.

Our SR technique for image processing plays a vital role in our high performance face detector. The algorithm works properly in case of dark images, but in case of bright images, the image equalization process is required for the image enhancement of dark images. The iteration number for the summation series is less in case of bright image than dark image.

The auto tuning process is required for fixing the iteration number to perform the summation of a given image. The darkness and brightness of an image plays a major role in the summation iteration number. The bright images are less iterated whereas, the dark images are iterated for several times. In case of the brightest image, the image has to be inverted at first and then only our SR technique has to be applied.

We will be working to create a system for auto-tuning the summation iteration number depending on the contrast and brightness of an image. The development of such a system will help us to make our system more effective.

\section{Conclusions}

In this paper, we presented a high performance face detector using our SR technique for image processing that works in the dark and illuminant variant images. The proposed algorithm enhanced the dark image and then detects the presence of faces in an image.

The environmental condition of dark and illumination variant images for face detection will be solved by our SR technique based face detector. The role of stochastic resonance played a vital role in determining the 


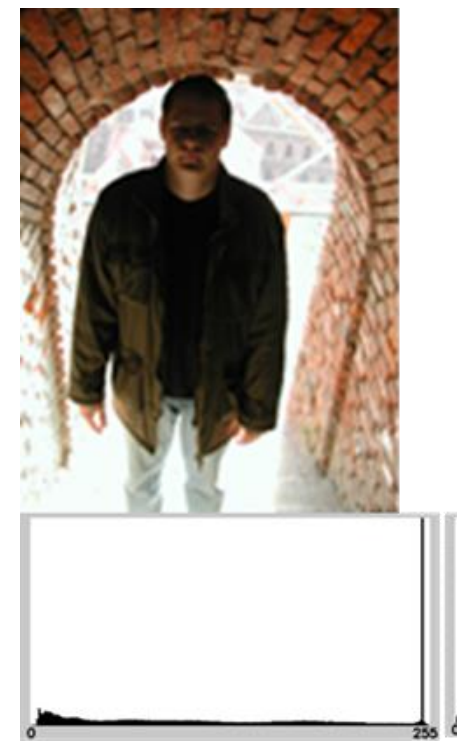

(a) Original image (from Ref. 8).

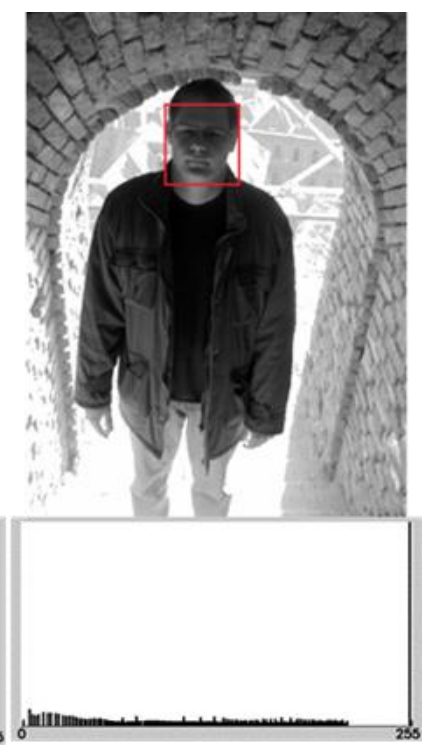

(b) Face detection after contrast adjustment
Fig.10. Evaluation on a case image taken inside a tunnel.

development of our high performance face detector.

The effects of stochastic resonance as our new approach was properly evaluated and experimented on different dark and illumination variant images with the appropriate results. The presence of a face, either in a dark or bright condition was detected by using our face detector along with our SR technique.

This proposed face detector based on our SR technique will help to increase the face detection rate on dark and illumination variant images as well.

\section{References}

(1) Bikash Lamsal and N. Matsumoto: "Effects of the Unscented Kalman Filter process for high performance face detector", Intl. J. of Information and Electronics Engineering, Vol. 5, No. 6, pp. 454-459, Nov. 2015 (To be published).

(2) T. Sim and T. Kanade: "Combining models and exemplars for face recognition: An illuminating example", In Proceedings of workshop on models versus exemplars in computer vision, CVPR 2001.

(3) S. Hayashi and O. Hasegawa: "Face detection in low resolution images", ISVC'05 Proc. of the first intl. conf. on advances in visual computing, pp. 199-206, 2005.

(4) F. Moss, L. M. Ward, W. G. Sannita: "Stochastic resonance and sensory information processing: a tutorial and review of application", Clinical Neurophysiology, vol. 115, pp267-281, 2003.
(5) R. Benzi, A. Sutera and A. Vulpiani: "The mechanism of Stochastic Resonance", J. of Physics, A: Math. and General, vol.14, pp. L453 -L457, 1981.

(6) R. K. Jha, P.K. Biswas and B.N. Chatterji: "Image denoising using Stochastic Resonance", Proc. of the Intl. Conf. on Cognition and Recognition, pp. 343-348.

(7) J.J. Collins, C. C. Chow and T. T. Imhoff: "Stochastic resonance without tuning", Letters to NATURE, Vol. 376, pp. 236-238, 1995.

(8) INRIA Persons Dataset, the EU project LAVA (IST2001-34405) and the Austrian Science Foundation (project S9103-N04).

(9) V. Jain and E. L. Miller: "FDDB: A benchmark for face detection in unconstrained settings", Technical Report UM-CS-2010-009, Dept. of Computer Science, Univ. of Massachusetts, Amherst, 2010.

(10) S. Kasai, K. Miura and Y. Shiratori: "Thresholdvariation-enhanced adaptability of response in a nanowire field-effect transistor network", Applied Physics Letters 96, 194102, 2010. 